\title{
Polarimetry of light scattered by surface roughness and textured films and periodic structures in nanotechnologies: a new challenge in instrumentation and modeling
}

\author{
F. Ferrieu.
}

STMicroelectronics, 850, rue Jean Monnet, F38926 CROLLES Cedex France.

\begin{abstract}
Exhaustive studies in the literature detail the Mueller matrices properties through decomposition, optical entropy and depolarization formalism. It has been applied for many years in rather different fields. In radar polarimetry, mathematical basis of depolarizing systems, have been first developed. In the visible range optics, standard diattenuation and retardance, decomposition is currently used in turbid organic media. The optical entropy concept, developed by S.R. Cloude, provides a very powerful analysis technique yielding important surface parameters such as depolarization, correlation and roughness. Complementary applications exist in scatterometry, for thin periodic grating films. With high capability polarimeters, such as the next generation of angle resolved polarimeters instruments, Polarimetry opens new fields of investigation for nanotechnologies materials as well as for gratings and photonics structures analysis: a program presently developed through a national consortium ANR08-NANO-020-03. With this instrumentation progress, simulation remains a key point to overpass as a challenge between future instruments. The theories for surfaces spectral power density (PSD) and the random coupled wave approximation (RCWA) in periodic structures are widely described in the literature. The implementation of some of these codes is described here for surface analysis and lithography scatterometry structures: grating overlay or double patterning.
\end{abstract}

\section{Introduction}

Exhaustive theoretical studies describing the role of Mueller matrices in Polarimetry are applied to many domains such as nanotechnologies, surfaces, earth sciences, astrophysics, thin films interface properties, surface roughness, photonics items, periodic gratings, diffracting objects and even living organisms in medicine. The polarimetry field began to be investigated with the earlier amplitude division polarimeters [1], but a large extent in imaging polarimetry is continuously growing. The variable angle spectroscopic ellipsometers (VASE) systems are being equipped with rotating compensators in order to achieve a full Mueller matrix measurement capacity. But today's Polarimeters have specific enhancements in both precision and speed. They use no moving parts which is a gain in highly reduced acquisition time [2,3] and stability. The high capability of today's polarimeters is revealed in new generation of angle-resolved instruments and potential interferometer capability. Behind this instrumentation progress, simulation, remains still a key point to overpass and a challenge.

Presently surface state characterization analysis is generally done considering only diffracted light intensities and through the classical surface power spectral density (PSD) analysis. To another extent, Mueller polarimetry reveals much more accuratel surface roughness potential. The pioneer works has been accomplished by Leroy-Brehonnet [4], In some cases the former effective medium approximation (EMA) theory remains not sufficient when processing accurate SE data. Depolarization effects can be observed when refining models. Reported $[1,4,5]$ data of Mueller matrices for surfaces with different roughness corroborate these observations.

In the classical polar decomposition, any Mueller matrix $[\mathrm{M}]$ can be seen as the product of depolarization, retardance and diattenuation. Complementary, the S.R. Cloude [6] "spectral" decomposition, describes a 


\section{EPJ Web of Conferences}

different understanding. Introducing the Bernoulli model provides together with the optical entropy formalism, a very powerful intrinsic analysis corresponding to stochastic systems.

Several examples from the Nanotechnologies materials analysis are given here and discussed in a first part of this paper. Then we focus to the periodic structures, comparing existing diffraction software codes to simulate polarimetric properties to demonstrate the interest of the technique.

\section{Non Depolarizing or depolarizing surfaces: The S.R. Cloude analysis}

\section{.2.1 A Way to Han dle the Mueller Matri Representation}

All the Mueller matrix elements $[\mathrm{M}]=\left[\mathrm{m}_{\mathrm{ij}}\right]$ are given, using the development of a coherency matrix [T] This coherency matrix is defined with elements given by a temporal average correlation between each Stokes vectors components. In an earlier papers, S.R. Cloude [6,7], demonstrated the SU(2) to SU(4) Lie's groups homorphism associated to $[\mathrm{T}]$ and $[\mathrm{M}]$. Briefly speaking, the coherency matrix $[\mathrm{T}]$ has to be set under the parametric form:

$$
[T]=\left[\begin{array}{cccc}
A_{0}+A & C-i D & H+i G & I-i J \\
C+i D & B_{0}+B & E+i F & K-i L \\
H-i G & E-i F & B_{0}-B & M+i N \\
I+i J & K+i L & M-i N & A_{0}-A
\end{array}\right]
$$

All the coefficients $\mathrm{A}, \mathrm{A}_{0}, \mathrm{~B}, \mathrm{C} \ldots \mathrm{N}$, are given as all real quantities, (here, $\mathrm{i}$ is the imaginary symbol). The corresponding Mueller matrix $\mathrm{M}$ is

$$
[M]=\left[\begin{array}{cccc}
A_{0}+B_{0} & C+N & H+L & I+F \\
C-N & A+B & E+J & K+G \\
H-L & E-J & A-B & M+D \\
I-F & K-G & M-D & A_{0}-B_{0}
\end{array}\right]
$$

The eigen values and eigen vectors decomposition of $[\mathrm{T}]$ will be 4 positive real values corresponding to an unique propagation Stoke vector $\mathbf{k}$. This decomposition theorem in 4 target-components, are each associated with the eigen values $\lambda_{\mathrm{j}}$ of the Hermitian coherency matrix [T] such as

$$
[M]=\sum_{i=0}^{3} \lambda_{i}\left[M_{i}\right]
$$

\subsection{Case of a random media and the stochastic hypothesis}

When the stochastic nature of a random media is considered, a mean measured Mueller matrix $<[\mathrm{M}]>$, is built over the eigen values of the average coherency matrix $<[\mathrm{T}]>$. Each eigen vector of $\langle[\mathrm{T}]>$ is representing one of the possible states with the probabilities $\mathrm{p}_{\mathrm{i} . .}$ The randomness is then characterized, with the Neumann entropy $\mathrm{S}$, such that, $0 \leq \mathrm{S} \leq 1$, if one has:

$$
S=-\sum_{i=1}^{N} p_{i} \log _{N} p_{i}
$$

where the $p_{i}$ are given by

$$
p_{i}=\lambda_{i} / \sum_{j}^{N} \lambda_{j}
$$

In the Bernoulli model, a parameter $\alpha$, is introduced as the following average:

$$
\overline{\bar{\alpha}}=\sum_{i}^{N} p_{i} \alpha_{i}
$$

has to be evaluated. The interpretation of $\alpha$, is an estimate of the system average scattering mechanism or in other words, its corresponding the entropy S: For an isotropic depolarizer, the Mueller matrix is diagonal. The corresponding coherency $<[\mathrm{T}]>$ is also diagonal: In the [entropy $S, \alpha$ ] space, it will turn in term of a zero $4 \times 4$ matrix with the only non zero elements equal to $1, \mathrm{~m}, \mathrm{~m}$. Each probability $\mathrm{p}_{\mathrm{i}}$ can be determinate and yields the analytical value of the entropy $S$.

In a complementary approach [4], the depolarization factor (depol), can be express in term of $\mathrm{m}$. In the isotropic case, the $[S, \alpha]$ space is transformed into an entropy versus, depolarization space, i.e. $\left[S, p_{D}=1\right.$-depol] The $m$ value from S.R. Cloude $[7,8]$, has been replaced by $p_{D}=1-d e p o l$, a parameter which can be deduced from the depolarization measurements. It yields [4],

$S=-\left(3 p_{D}+1\right) / 4 \log _{4}\left(\frac{3 p_{D}+1}{4}\right)-3\left(1-p_{D}\right) / 4 \log _{4}\left(\frac{1-p_{D}}{4}\right)$

According to eq.(8), A. Aiello, J.P. Woerdman [9], predicted the universal behaviour of these quantities. Various experimental data, can be reviewed now by reconsidering this coherency matrix decomposition theory

\subsection{Experimental}

A first control can be accomplished with literature available Mueller matrix data, Ramsey, Leroy-Brehonnet, Azzam $[5,4,1]$, for various surface materials, e.g., Dielectric S, Nylon and Dielectric C[4]. All these values are perfectly aligned on the universal entropy depolarization relation given by eq (8), as shown also in figure 1 . The data of $B$. Boulbry [9] provided the case of polished ( 0.3 microns) aluminium (Al) sample). Even in this case when a first filtering has been accomplished, gives $\mathrm{S}=0.07$ and $\mathrm{p}_{\mathrm{D}}=$ 0.9930 , 


\subsubsection{Thin Films surface Characterization}

Within thin films surface characterization of nanotechnologies materials, spectroscopic polarimetry specifies surface properties and films textures. Several nanotechnologies material films were measured with the AutoSe polarimeters [2] (Horiba Sci.), from smooth samples like aluminum (Al), to low reflectivity thin films of plastic (PET), or Silicon Nano-Wires (SiNW) deposited on a silicon substrate. As shown in figure 2, together with the analytical equation (8), in the $\left[\mathrm{S}, \mathrm{p}_{\mathrm{D}}\right]$ space, the $\mathrm{Al}$ data (filled triangles), remain in a very high reflectivity limit $\left[5=0, p_{d} \sim 1\right]$

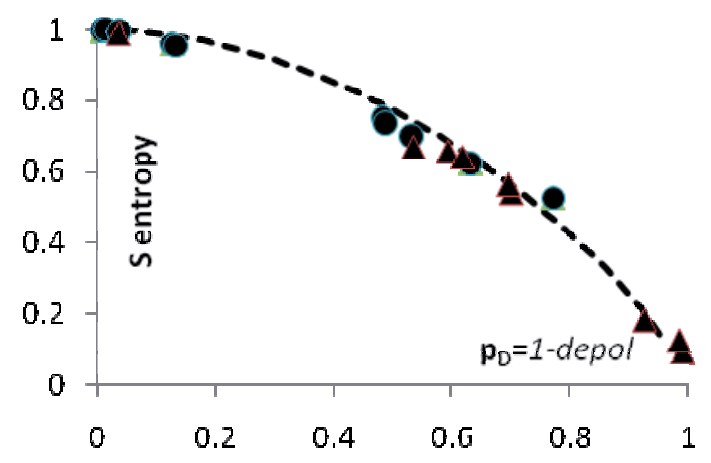

Fig. 1 Showing Entropy/depolarization space The Collected data correspond to various references sources $[3,4]$, including data from Ramsey[5], for aluminum surfaces wth roughness ranging from 1.2 microns down to $100 \mathrm{~nm}$. The dash line is equation( $(8)$.

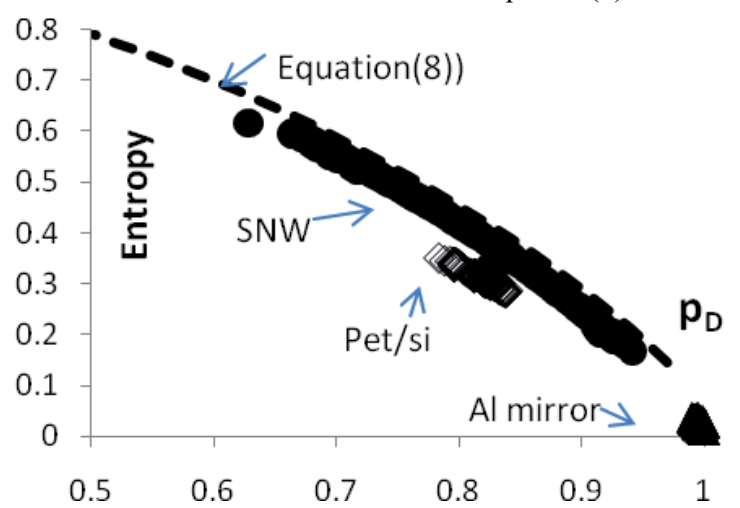

Fig.2 Entropy versus depolarization $\mathrm{p}_{\mathrm{D}}$ plot for several nanotechnology materials: with a $70^{\circ}$ incidence angle polarimetry spectroscopic measurements in the 1.5 and $3 \mathrm{eV}$ range, The respective spectroscopic distribution (filled dots, squares, triangles),follows well the universal character curve (eq. (8)) see details in text)

The SiNW film are considered as a distribution of micro crystals components, (needle-like and various shaped nanocrystals), turning to nano-sized light-scatterers, as revealed by Scanning Electron Microscopy, SEM, observation see figure 3 . In figure 2 , the $\left[S, p_{D}\right]$ space shows a "spectroscopic" distribution (filled dots) of SiNW sample following surprisingly well, the universal character curve from A. Aiello and J.P.Woerdmann [9].

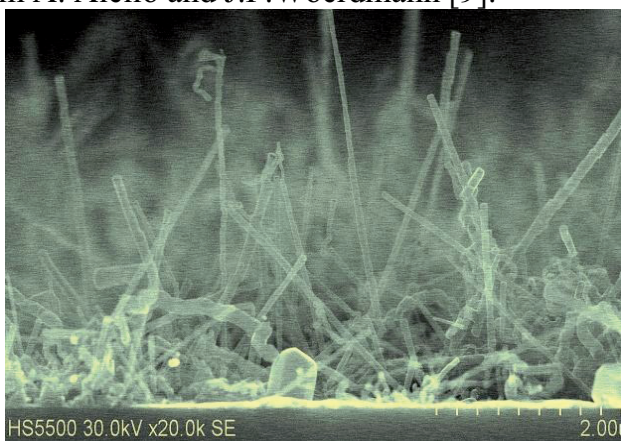

Fig.3 Scanning Electron Microscopy picture taken of silicon nano wires (SNW)deposited films (with courtesy of V. Jousseaume LETI), The $\left[\mathrm{S}, \mathrm{p}_{\mathrm{D}}\right]$,space representation all along eq(8)curve (see fig.2) is a perfect method to measure the microcrystal size distribution density of these films.

In that spectral range, the material optical indices remain quasi constant. Thus each point can be related to one possible entropy state of the system corresponding to a specific diffracting target at one wavelength. In another hand, considering the number of times these points appeared, all along this curve can be seen as a method to yield the micro crystals distribution density, Further investigations should still be done to clarify that.

In the entropy space $\left[S, p_{D}\right]$ points should be below the analytical curve, (eq(8)). Equation (8) remains the upper limit of the couples $\left(\mathrm{S}, \mathrm{p}_{\mathrm{D}}\right)$, standing for isotropic depolarization [4], i.e., the case of most analyzed samples here. The anisotropic plastic PET film, having to a less extent micro impurities, behave thus as expected (filled squares) below eq.(8).

\subsubsection{Instrumental accuracy control through}

Another interesting approach, consist in the intrinsic analysis of a spectroscopic polarimeters instrument itself. While placing it in $90^{\circ}$ (open-air or free-space measurement) configuration, no depolarization occurs and the entropy should be kept to zero. The depolarization index has to be always unity (a pure Mueller Stoke system). In this considered spectroscopic range the polarimeter fidelity can be checked so far. The physical realizability of experimental Mueller matrix for each wavelength can be then tested with this method. It is known as the Mueller matrix filtering technique, used to qualify the instrument purity, (usually of the order or more than -20 decibel differences [10]).

\subsection{The need for a Polar imetric Surface Scattering model: Slope distribution roughness}

Roughness surface disturbance can be considered by reflection symmetric, depolarizing stochastic rotations of 


\section{EPJ Web of Conferences}

the specular coherency matrix about an angle $\beta$ in a plane perpendicular to the scattering plane (slope distribution). A surface configuration average over all possible slopes angles value as a distribution $\mathrm{P}(\beta)$, has to be accomplished. S.R. Cloude $[6,7,8]$ proposed a slopes distribution uniform with half width $\beta_{1}$, and a probability $\mathrm{P}(\beta)=1 / 2 \beta_{1}$ when $|\beta|<\beta_{1}$, and zero otherwise. Then the $<\left[\mathrm{T}_{\mathrm{ij}}\right]>$ elements appears as

$$
\left\langle\left[T_{i, j}\right]\right\rangle=\int_{0}^{2 \pi}[T(\beta)] P(\beta) d \beta
$$

Only in this case, the polarimetric coherency correlation $\gamma$, defined as

$$
\gamma=\left\langle T_{12}\right\rangle / \sqrt{\left\langle T_{11}\right\rangle\left\langle T_{22}\right\rangle}
$$

becomes

$$
\gamma=\operatorname{sinc}\left(2 \beta_{1}\right) / \sqrt{1+\operatorname{sinc}\left(4 \beta_{1}\right)} \leq 1
$$

where $\sin c(x)=\sin (x) / x$. The $\gamma$ coefficient is shown to be only related to the surface roughness and not to the dielectric properties of the considered surface. This can be verified in some cases, e.g., for a $70^{\circ}$ incidence specular reflection on both sides of a Silicon wafer (polished side with native oxide and rough backside of the wafer, which is shown in figure 5 Notice that the roughness of a standard backside wafer ( $\sim$ several microns), is definitely higher than the order of the visible light wave length used by the instrument.

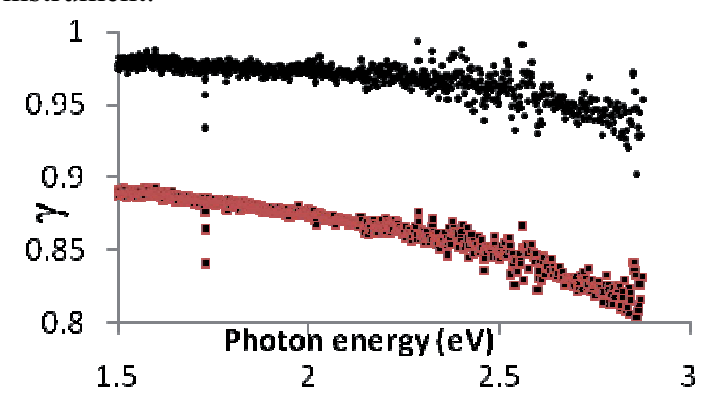

Fig. $570^{\circ}$ incidence $\gamma$ measurements between 1.5 and $3 \mathrm{eV}$ photon energies on both sides of a silicon wafer (polished side with native oxide (top) and rough backside of the wafer (below).

Here, the roughness controls entirely the $\gamma$ coherence factor, when no direct depolarization effect can be measured: SE measurements would provide only a shift in the $\Delta$.values and no noticeable change in $\Psi$. It can be concluded that only the $\tilde{\gamma}$ coherence factor. is the primary parameter to be considered.

\section{Periodic Structures}

For years, most applications of polarimetry have been dedicated in microelectronic to periodic structures, (the lithography gratings patterns). Scatterometry characterization is a routine in-line tool for etched lithography patterning, as these depicted in figure 6: transistor, grating lithography-etched (top of figure 6), but also in nanotechnology 2-dimensional (2D) organized planar materials, e.g.. as pillars 2-D distribution sample.( bottom of figure 6). Several codes describing diffraction of light by these structures exist such as the open source $\mathrm{C}^{++}$ of the National Institute of Standard and Technologies (NIST)[11] library or MatLab softwares.

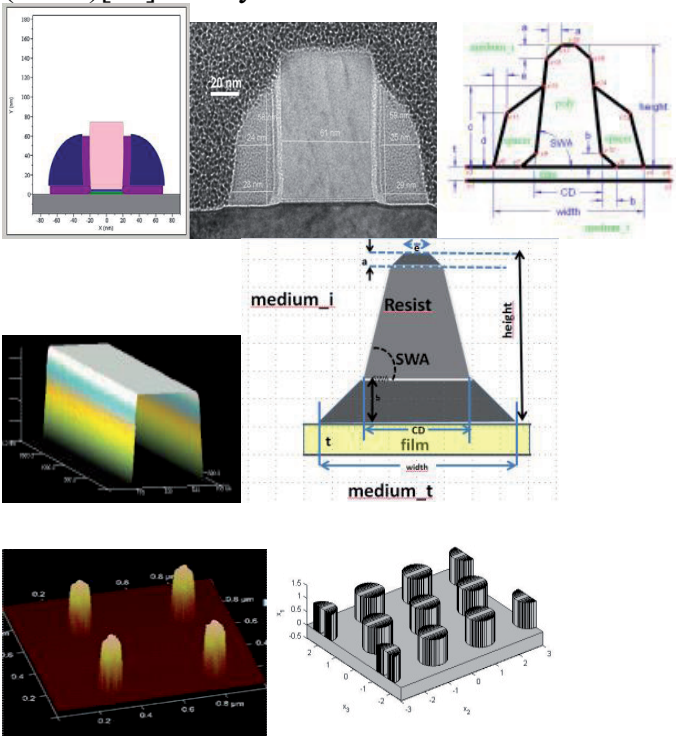

Fig 6 Typical structures of the microelectronic pattern lithography, first line from left to right, represent the structure decomposition of a transistor embedded between spacer, next is the corresponding TEM pictures and to the right side the scheme corresponding to the NIST[11] script file., Below a similar description of lithography grating pattern, as measured with AFM 3D (left), together with the scheme of the NIST script equivalent to a grating with rounded-corner shape with an intermediate stopetching resist(right). This is: a useful scheme when writing the script files of the $\mathrm{C}^{++}$NIST library. At the bottom is the picture retrieved from AFM 3D measurements (in the case of a $2 \mathrm{D}$ pillars structure for nano-imprints, and the corresponding model drawing for scattering (courtesy of LETI).

\section{.3.1 The NIST Scatmech $\mathrm{C}^{++}$library.}

The Scatmech code, (present 6.01 version ), is a RCWA C ++ code which can be downloaded, together with a user friendly MIST demo program. All the variables such as, dielectric function, Stokes vectors, as well as Jones and Mueller matrices are $\mathrm{C}^{++}$classes, which can instancied as objects. Furthermore, several types of gratings belong to the RCW class. The user can then access parameters with classical pointer assignments or setting up a script file describing the periodic structure. As shown in figure 7, for example, use of the rounded-corner grating RCW class easily fit the SE classical $\alpha, \beta$, data measured from either the AutoSe as well with most of the commercial 
SE[12] system. To optimize the experimental structure parameters, a complete optimization can be done with the $\mathrm{C}++$ non linear optimization routine $[13,14]$

For more complicated stacks, a script file is preferable. It can be filled following the design such as those from figures 6 . The script file could be adapted to general cases such as multi stacks or as shown here, also figure 8 , a double patterning process:
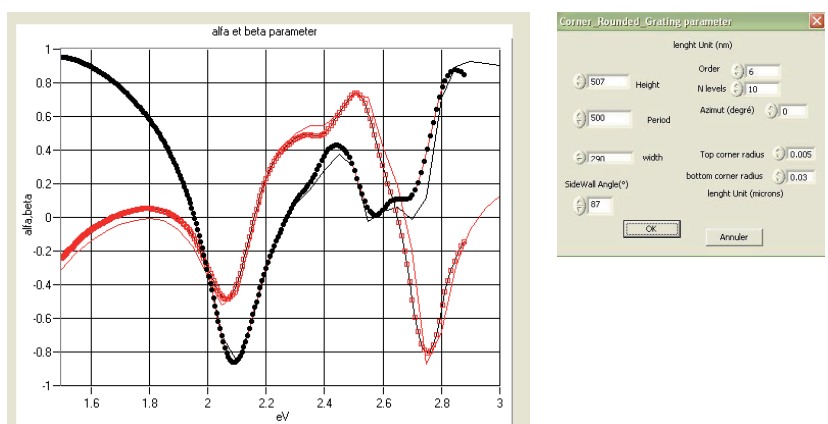

Fig 7. Use of the rounded corner grating properties of the RCW $\mathrm{C}^{++}$class from Scatmech $\mathrm{C}++$ library optimize the $\alpha, \beta$ SE parameter inside an optimization software[13,14].

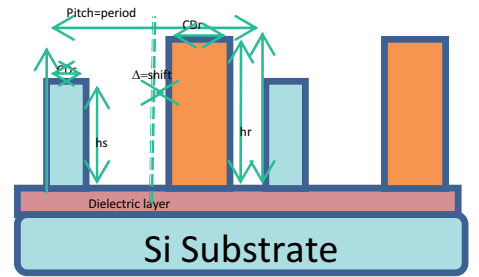

Fig 8 Double patterning figure to be used when building the script file inside the RCW class in the Scatmech software [11].

\section{A new Instrument: the Mueller Fourier angle-resolved Polarimeter.}

\subsection{The Instrument}

Beside its full capabilities, this polarimeter designed in the LPICM laboratory [3] has an optical setup reconfigured within a microscope geometry, light source LS, sample S, mirror $\mathrm{M}$ or dispersive objective, Objt, (fig.9), enabling simultaneous collection of different incidence and azimuth angles while imaging the Fourier plane of the microscope on the CCD F plane [15]

.Typically, Mueller matrices are obtained in their $4 x 4$ matrix representation each element corresponding to the simultaneously collected angles. This setup does not enable yet a full spectroscopic investigation but only few wavelengths due to the polarization state generator and analyzer units (PSG, PSA) and objectives limits. Therefore only one or two wavelengths can be selected. However angular resolution remains high as from normal incidence, (zero) to $70^{\circ}$ degrees incidence and zero and 0 to $360^{\circ}$ azimuth capability. This instrument has a much more adapted spot size (some few microns against tenth of microns in the case of a Variable Angle Spectroscopic Ellipsometer (VASE) instrument.

Courtesy of LPICM A. De Martino and

Ben Hatit et al.: phys. stat. sol. (a) 205, No. 4 (2008)

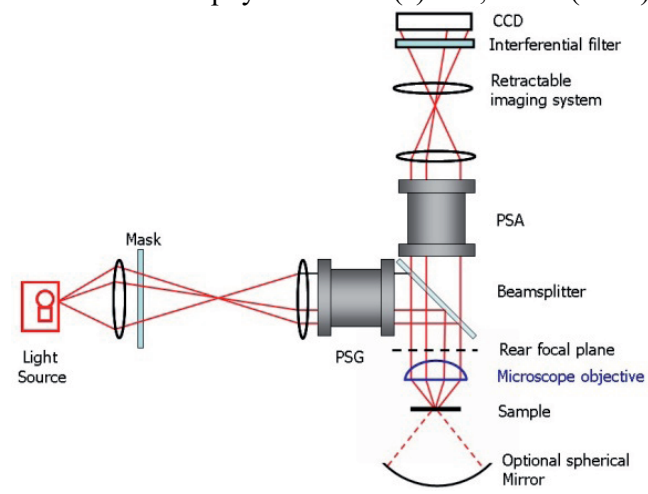

Fig 9 The angle resolved Mueller Fourier polarimeters developed by LPICM and in the ANR08 NANO-020-01 see reference [3]. Equivalent in-line metrology equipment is currently under design by Jobin Yvon Horiba Sci., partner in the ANR08 NANO-020-01 consortium with LETI.

\subsection{The Optimisation Software}

LPICM develops its own undisclosed software but comparison with Scatmech or open source codes can be made and agree fairly well. Available MathLab codes exist even they are calculating only light intensities efficiencies from the scattered electric field components. Implement of the classical the Mueller matrices calculation from the scattered electric field components has been carried out for comparison. Similar to the drawing given, see the figure 8 , the case of a silicon grating can be recalculated easily.
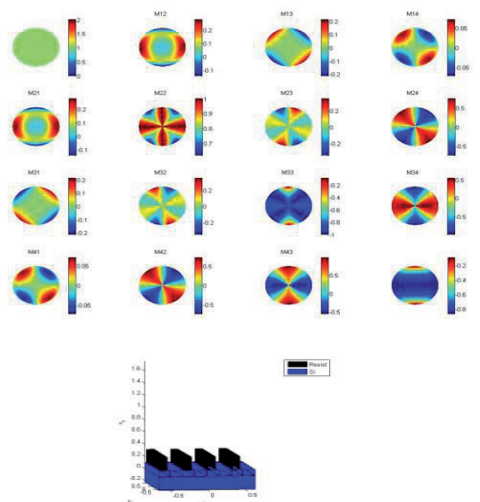

Fig 10 Angle resolved Mueller Fourier simulation in the case of a Silicon grating (CD- $150 \mathrm{~nm}$, pitch 300 and height $500 \mathrm{~nm}$ )

Also, as demonstrated in figure 11, 2-D pillar lattice (schematic drawing) is reported with the corresponding angle resolved Mueller Fourier simulation (at top of fig.11). 3-D (photonic) structures could be investigated as well (see drawing and corresponding simulation result (bottom)). 
This shows simply that polarimetry will be the appropriate tool in a next future to solve these structures

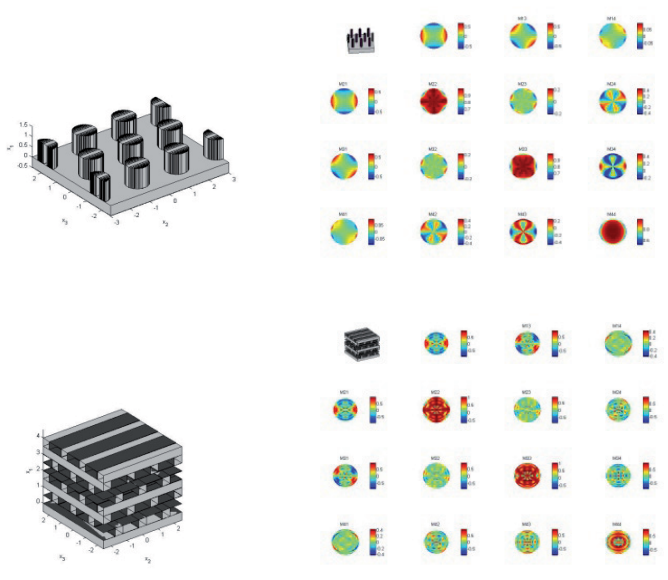

Fig 11 Angle resolved Mueller Fourier simulation, (top) in the case of 2-D (pillars) and cross linked gratings (3-D) structures (bottom)

\subsection{Discussion and conclusions}

The Mueller matrices decomposition scheme provides interesting properties through optical entropy and depolarization. This technique is a good candidate to yield important surface parameters such as depolarization coherence, entropy and furthermore surface roughness characteristics very promising for future nanotechnologies. In that sense, Spectroscopic polarimetry should be addressed to the surface properties of deposited films inline processes control. Beyond the scope of this paper, a visible ultraviolet-extended spectroscopic range of the existing polarimeters set-up, including also the imaging and multi-angle resolved capability, would bring many benefits as compare to present only bidirectional reflectance distribution function (BRDF) and haze ultraviolet wafer classical analysis of wafers.

Non destructive, Scatterometry probes only SE parameters, corroborating perfectly other destructive characterization techniques. However, due to possible parameters correlations, it yields some times, evasive conclusions. These effects are considerably reduced with angle resolved Mueller Fourier polarimetry. Beside this fundamental aspect, a much more reduced spot size (few microns) and the multi-angle capability make this new instrument definitely very promising. Software sources of the SE metrology have to move to full Mueller matrix optimization in a near future. By simulating the structure and design of periodic stack and organized structures, the existing Code such as SCATMECH library or MATLAB sources are already useful in the knowledge of the optical properties of new nanotechnology materials which is a key point in the future of nano-photonics developments

\section{Acknowledgements}

This work is supported by the French National Research program ANR-PNANO2008 MUELLERFOURIER with the consortium of the LPICM, Horiba Sci. and LETI 20092011.

The author thanks for efficient discussions with the ANR participants from LPICM / A. de Martino, B. Drevillon and LETI / C. Vanuffel and J.C. Royer..

\section{References}

[1] R.M.A. Azzam and N.M. Bashara, "Ellipsometry and Polarized light" p 156-157 NH Elsevier 1996

[2] www.horiba.com and see also www.axometrics.com

Hind Co at www.hindspem.com see also ref [12]

[3] Ben Hatit Thesis Ecole Polytechnique Paris(2005) and Ben Hatit, M. Foldyna,A. de Martino, and B. Drevillon Phys. Stat. Solidi (a) 205,4 (2008).

[4]F. Leroy-Brehonnet et al, J. Phys. D: Appl. Phys.29, 34-38,(1996).

[5] D. Ramsey, K .Ludema Rev. Sci. Instrum. 65, (9) 2874, (1994)

[6] S.R. Cloude SPIE 3754 (july 1999)

[7] S.R. Cloude, E. Pottier Opt. Eng.34, 6, 15991610(1995) and references therein

[8] S.R. Cloude SPIE, 3121,88-99.

[9] A. Aiello, J. P. Woerdmann, Phys. Rev. Letters, 94, 090406, (2005).

[9] F. Boulbry thesis University Bretagne, (2002)..

[10] F. Stabo, M. Kildermo, I.S. Nerbo, M.

Lindgren, Opt. Eng. 47(7),073604-(1-9)],(2008).

[11] /physics.nist.gov/Divisions/Div844/facilities/

[12] www.kla.com or www.sopra-sa.com

[13] http://www.nr.com/com/storefront.html

[14] www.chem.polimi.it/homes/gbuzzi

[15] A. de Martino US20070263219. 